Revista de Psicología de la PUCP. Vol. XXII, 2, 2004

\title{
Meta-análisis sobre estudios de identificación y atención al talento y la superdotación en el Perú
}

\author{
Sheyla Blumen de Pardo ${ }^{1}$ \\ Pontificia Universidad Católica del Perú
}

\begin{abstract}
¿Tienen los programas de identificación del talento y la superdotación bases científicas sólidas, justas e imparciales? ¿Son los programas de intervención para talentosos y superdotados efectivos? Las presiones financieras que ejercen las fundaciones que brindan apoyo económico, los cambios recientes en las leyes, así como las consideraciones respecto a la multiculturalidad están forzando a los psicólogos y educadores alrededor del mundo a confrontar estos cuestionamientos. La presente revisión discute los cambios que han empezado a alterar este campo de estudio en América Latina y en especial en el Perú. Se presenta un análisis de estudios empíricos en poblaciones multiculturales, así como una reflexión sobre el impacto de los programas de intervención, a nivel de capacitación docente y de enriquecimiento cognitivo/afectivo, que se desarrollan en la región.

Palabras clave: talento, superdotación, multiculturalidad, identificación, enriquecimiento, diversidad.
\end{abstract}

Meta-analyses on studies about the identification and attention of the gifted and talented in Peru

Are gifted and talented identification programs scientifically based, fair and unbiased? Are gifted enrichment programs effective? Financial pressures from NGOs and private foundations, changes in national educational policies, as well as multicultural issues, are forcing psychologists and educators to face these questions. Our review discusses the important changes that have begun to alter the gifted and talented field in Latin America, especially in Peru. It describes recent empirical studies in multicultural populations and analyses the impact of the intervention programs, both, on the teacher training level as well as on the gifted enrichment programs, which are being developed in the region.

Key words: high ability, giftedness, multiculturality, identification, enrichment, diversity.

1 Profesora Asociada de Psicología en la PUCP. Representante del European Council for High Ability para Perú/América Latina y Coordinadora del Diploma ECHA en el Perú. Psicóloga supervisora a nivel Perú de MENSA International. Directora de Mente Futura, Centro de Investigación en Creatividad y Talento. Correo electrónico: sblumen@pucp.edu.pe 

La niña y el niño talentoso es víctima de la incomprensión de sus compañeros y maestros en el aula de clases y de sus padres y hermanos en el ambiente familiar. Los esfuerzos de sensibilización respecto a las necesidades de los talentosos y superdotados realizados por diversas organizaciones internacionales, tales como el Consejo Mundial de Superdotación y Talento (World Council for the Gifted and Talented Children) y los consejos regionales vinculados a éste (i.e. FICOMUNDYT) y el Consejo Europeo de Altas Capacidades (European Council for High Ability), entre otros, son insuficientes dado que favorecen principalmente a aquellos que tienen mayores posibilidades de acceso a sistemas educativos de calidad. Más aún, la condición actual de la investigación en talento y superdotación presenta dificultades meta-teóricas y ontológicas respecto a la concepción de estos constructos, lo cual dificulta sus posibilidades de generalización a poblaciones multiculturales.

Fuerzas económicas, culturales, políticas, legales y científicas están causando cambios profundos en las prácticas de identificación psico/educacional. En primer lugar, las presiones financieras de los servicios de atención, las instituciones educativas y la necesidad de justificar el porcentaje de talentosos identificados frente a las fundaciones privadas que brindan apoyo económico, han alterado las prácticas de identificación de numerosos psicólogos y educadores. En segundo lugar, desarrollos recientes en las leyes educativas de apoyo a la integración de la diversidad, están forzando el análisis crítico hacia las técnicas de identificación que son utilizadas en ámbitos clínicos y educativos.

En tercer lugar, la composición étnica de los países latinoamericanos presenta nuevos retos hacia las viejas prácticas, 
dado que los niños aborígenes o nativos tienden a presentar una participación limitada en los programas para superdotados y/o talentosos. En este sentido, existen fuerzas internas y externas a nuestra área de estudio que están proponiendo los siguientes cuestionamientos: ¿son las evaluaciones psicológicas de identificación del talento y la superdotacion justas e imparciales? ¿presentan una base científica clara? Las respuestas podrían reformular la ciencia y la práctica de los procesos de identificación de niños talentosos en desventaja cultural.

\section{Evaluación multicultural}

En la composición étnica de los países latinoamericanos se observa que, si bien algunos de éstos presentan cambios acelerados en su proceso de occidentalización, otros países como Ecuador, Perú, Bolivia y Brasil mantienen aún su condición multicultural y multilingüe, que comprende alrededor de 40 lenguas vernaculares que se hablan en la región amazónica, fuera del Aymara en la región del altiplano y de las diferentes versiones del Quechua que aún se mantienen en la región andina.

La atención hacia los programas de identificación en ámbitos multiculturales se ha incrementado en años recientes entre los académicos y los psicólogos orientados hacia la práctica, como lo evidencia la publicación de los artículos de Bechervaise (1996) y Keighley (1984) sobre este tema. Se observan también investigaciones de nivel descriptivo-correlacional y experimental desarrolladas en el Perú (Blumen, 1998, 2000) y la inclusión de tres simposios internacionales en el último XXIX Congreso Interamericano de Psicología desarrollado en la ciudad de Lima en julio del 2004 (Blumen, 2003), donde se presentaron estudios sobre las variables asociadas a los procesos de identificación y atención a niños y adolescentes talentosos, resaltándose la 
Meta-análisis sobre estudios de identificación y atención al talento.

importancia de la capacitación docente en esta área, así como la necesidad de marcos legales que favorezcan su aplicación. Cabe resaltar la importancia que tiene el que se haya aceptado la inclusión de simposios sobre este tema en un congreso internacional de psicología.

Pese a que se observa un avance en la región, la calidad de la investigación continúa estando lejana frente a lo que se necesita. Los estudios acerca de los talentosos y superdotados latinoamericanos son escasos y por lo general adolecen de técnicas de evaluación válidas para la población en estudio. En este sentido, la prueba de Pensamiento Crítico de Torrance (TTCT), el Test de Pensamiento Creativo-Producción Figurativa (TCT-DP) de Urban y Jellen (1994), el Test de Habilidades Cognitivas de Thorndike y Hagen (Cog At), Versión 5 (1993), el Psicodiagnóstico de Rorschach (Sistema Comprehensivo de Exner), el Test de Apercepción Temática (TAT) y el MMPI-2, entre otras pruebas, están aún en proceso de estudio a nivel de las características psicométricas para la población donde se pretenden aplicar. Asimismo, se necesita examinar las posibles diferencias en la validez de los puntajes de las pruebas a lo largo de grupos culturales y linguísticos diversos (Thorne \& Blumen, 1996).

Se observa la emergencia de nuevas formas de evaluación, complementarias a las existentes, como un intento de afrontar la falta de pruebas válidas y confiables para las poblaciones multiculturales. En este sentido, variables tales como la motivación intrínseca han sido evaluadas a partir del nivel de productividad con la técnica de Evaluación del Portafolio (Blumen, 2001), y el desempeño creativo, tanto a través de la producción figurativa como a través del cuestionamiento verbal, ha sido evaluado a través del Test de Pensamiento CreativoProducción Figurativa de Urban y Jellen (Blumen, 2000, 2003) y del Test de CREA (Blumen, 2004; Corbalán, Martínez \& Donolo, 2003), respectivamente. En los años recientes, el Inventario de 
Motivación Intrínseca de Logro para Niños (CAIMI) ha sido también considerado (Blumen \& Cornejo, 2004).

Los estudios normativos de las pruebas psicológicas desarrollados por Ardila y sus colegas (Ardila, Rosselli \& Puente, 1994), así como estudios con el Test de Matrices Progresivas de Raven (CPM) (Esquivel, 2000; Thorne \& Blumen, 1996) y los estudios normativos con el Test de Pensamiento CreativoProducción Figurativa (Alencar, Blumen-Pardo \& CastellanosSimons, 2000; Alencar, De Souza \& Blumen-Pardo, 2002; Blumen, 1998, 2000, 2003), han marcado un paso importante en la evaluación multicultural en América Latina. Estos estudios han identificado sistemáticamente variables moderadas, tales como el nivel educativo más que la edad cronológica, como significativas en los resultados de la evaluación de niños talentosos y superdotados latinoamericanos que viven en condición de desventaja cultural.

Otro desarrollo importante ha sido la publicación de algunas escalas de aculturación adecuadas a espacios multiculturales (Stephenson, 2000). Los instrumentos desarrollados recientemente conceptualizan la aculturación como dos dimensiones separadas más que una única dimensión bipolar. La aculturación es importante tanto en el trabajo clínico como en el educacional, debido a que en ocasiones tiende a moderar la validez de los puntajes de las pruebas (Cuellar, 2000).

Tomando en consideración las sugerencias de Ardila et al. (1994) respecto a la evaluación en ámbitos clínicos, cuando se desee identificar a niños talentosos y superdotados en ambientes multiculturales es importante evaluar de manera rutinaria el nivel de aculturación de la persona, las eferencias lingüísticas, así como la edad y el bagaje educacional, para luego proceder a seleccionar los instrumentos y las normas que serán más apropiadas para su evaluación (Blumen, 2003). 
Meta-análisis sobre estudios de identificación y atención al talento.

Factores asociados a la superdotación en niños de segundo grado de Lima

A continuación se analiza un estudio empírico realizado en el Perú que estudia los factores asociados a la superdotación en niños con desventaja cultural que cursan el segundo grado de primaria y viven en la ciudad de Lima (Blumen, 1998). Se conceptualiza la superdotación según la triada personal del Modelo Multifactorial de Superdotación de Mönks (1992) (ver Figura 1).

A partir de la pregunta de investigación: ¿cuál es la relación entre la identificación del superdotado con el Modelo Multifactorial de Superdotación y el desempeño escolar en estudiantes de segundo grado de un colegio público de Lima?, se definieron los siguientes objetivos:

- Determinar la motivación de los participantes a través del nivel de productividad intelectual.

- Caracterizar la creatividad a través del desempeño creativofigurativo de los participantes.

- Determinar la capacidad superior según el potencial intelectual de los participantes.

- Identificar la relación entre la productividad intelectual, el desempeño creativo-figurativo y el potencial intelectual.

- Determinar el nivel de rendimiento escolar de los participantes.

- Comparar la identificación del superdotado según la interrelación entre la productividad intelectual, el desempeño creativo-figurativo y el potencial intelectual, con el rendimiento escolar en segundo grado. 


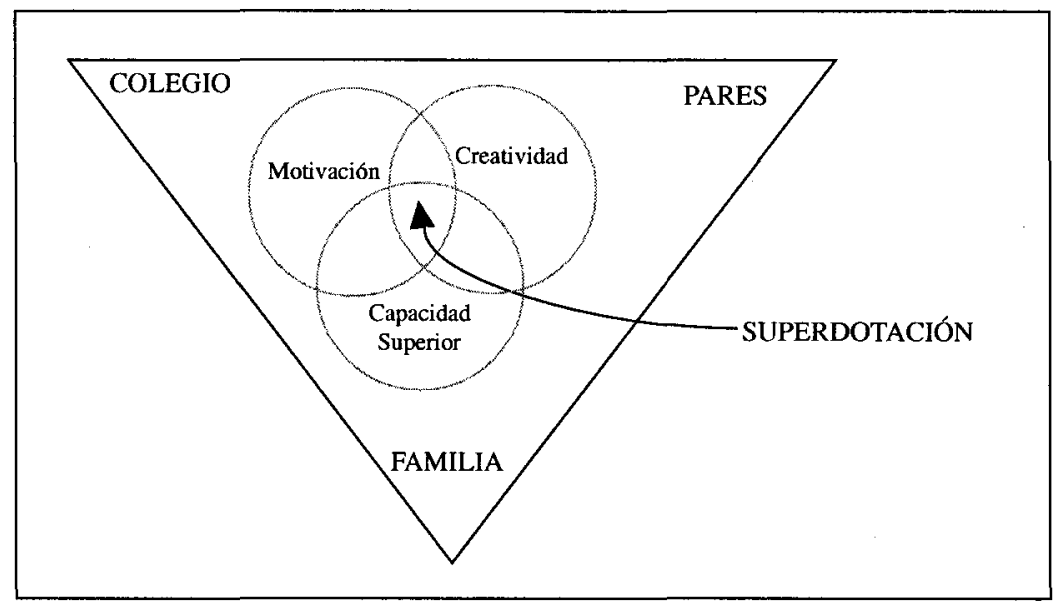

Figura 1. Modelo Multifactorial de Superdotación (Mönks, 1992).

Las variables del estudio fueron las siguientes:

- Productividad Intelectual, en función a los resultados del Método del Portafolio.

- Desempeño Creativo-Figurativo, evaluado según los resultados con el TCT-DP de Urban y Jellen.

- Potencial Intelectual, a partir de los resultados con el $C P M$ de Raven.

- Rendimiento Escolar, operacionalizado en base a las calificaciones del primer semestre.

El estudio fue de nivel descriptivo-correlacional, debido a que pretende especificar el nivel de productividad intelectual, desempeño creativo-figurativo y potencial intelectual de los participantes. Se examina también el grado de relación existente entre las variables.

El tamaño muestral se calculó considerando el número mínimo de unidades de análisis necesario para formar una muestra $(n)$ que garantice un error estándar menor que .01, considerando que la población $N$ es aproximadamente de 8000 
Meta-análisis sobre estudios de identificación y atención al talento.

elementos. El tamaño muestral fue de 217 casos, obtenidos a través de un muestreo probabilístico por conglomerados en dos etapas: en la primera etapa se seleccionaron 60 profesores de segundo grado que obtuvieron los mejores puntajes en el Plan de Capacitación Docente de 1996; en la segunda etapa se seleccionaron los participantes (40 alumnos por profesor), tomando en cuenta el tamaño de la muestra y el procedimiento de selección.

Este procedimiento permitió seleccionar 217 estudiantes de segundo grado en desventaja cultural de la ciudad de Lima, 106 superdotados (53 niñas y 53 varones) y 111 no superdotados (54 niñas y 56 varones). Tomando en consideración las características sociodemográficas de la muestra, los participantes vivían en áreas urbano-marginales, sus padres tenían niveles educativos primarios y estaban empleados en actividades artesanales, venta ambulatoria o eran trabajadores no calificados. En ambos casos, el rango de edad estuvo entre los 6 y 11 años, con una media de 7.4 y una mediana de 7.3.

Se elaboró una Escala de Observación para Padres (Blumen, 1998), así como una escala para evaluar la productividad del estudiante a través del Método del Portafolio (Blumen, 1998). Asimismo, se analizaron las características psicométricas de los siguientes instrumentos: Test Cog At de Thorndike y Hagen, versión 5 (1993), Test de Matrices Progresivas de Raven, versión Coloreada, y Test de Pensamiento Creativo-Producción Figurativa de Urban y Jellen, Forma B (1986). Se analizaron también los indicadores del sistema educativo peruano asociados con la superdotación.

Luego de solicitar el consentimiento informado de los padres y maestros de los participantes, y, tomando en cuenta las consideraciones éticas de la investigación psicológica con humanos, se procedió a la recopilación de datos y elaboración de la base de datos que incluyó variables sociodemográficas, 8 variables del Método del Portafolio, 15 variables del TCT-DP, 37 
variables del $C P M$ y 8 variables relacionadas con el rendimiento escolar. Fueron 75 variables ingresadas por participante.

El análisis de resultados consideró primero la prueba de Kolmogorov-Smirnov para evaluar la bondad de ajuste en la distribución normal de la muestra, en la que se observaron diferencias significativas en todas las variables estudiadas: Método del Portafolio $(K-S(210)=0.21, p<.001), T C T-D P(K-S(210)=$ $0.087, p<.001), C P M(K-S(210)=0.16, p<.001)$, y Rendimiento Escolar $(K-S(210)=0.21, p<.001)$. Por lo tanto, se consideró el uso de pruebas no paramétricas para la comparación de resultados.

El nivel de productividad intelectual se examinó a nivel descriptivo y comparativo aplicando la $U$ de Mann-Whitney a los resultados del Método del Portafolio, donde se observó que el grupo de los superdotados exhibió una mediana (Mediana $=$ $31.00, I . R=3.00)$ significativamente mayor que los no superdotados (Mediana $=16, I . R .=9.00), Z(215)=-12.46, p<$ .001 (ver Cuadro 1). También se observó que los no superdotados exhibían un mayor rango de dispersión en la distribución de puntajes, mientras que los superdotados presentaban una distribución más homogénea. Más aún, es importante observar que la frecuencia de distribución del grupo no superdotado presentó curva bimodal, mientras que la distribución de los superdotados presentó sesgo negativo.

\section{Cuadro 1}

Diferencias entre los puntajes del Método del Portafolio

\begin{tabular}{lcccc}
\hline & No superdotados & Superdotados & $U$ & $Z$ \\
\hline $\begin{array}{l}\text { Mediana } \\
\text { Rango }\end{array}$ & 16 & 31 & 138 & $-12.46^{* * *}$ \\
$\begin{array}{l}\text { Intercuartil } \\
\text { Rango de la }\end{array}$ & 9 & 3 & & \\
Media & 56.75 & 162.71 & & \\
$n$ & 110 & 107 & & \\
$* * * * p<.001$ & & & &
\end{tabular}


Meta-análisis sobre estudios de identificación y atención al talento.

En el desempeño creativo-figurativo los resultados muestran que la mediana de los superdotados (Mediana $=15, I . R .=6)$ fue significativamente mayor que la mediana del grupo de los no superdotados (Mediana $=9$, I.R. $=6), Z(211)=-8.69, p<.001$, presentando la distribución de puntajes similaridad entre ambos grupos (ver Cuadro 2). La distribución de frecuencias del grupo no superdotado fue mesocúrtica, sesgada positivamente, mientras que la distribución del grupo superdotado presentó una curva con sesgo negativo.

\section{Cuadro 2}

Diferencias entre los puntajes del test TCT-DP

\begin{tabular}{lcccc}
\hline & No superdotados & Superdotados & $U$ & $Z$ \\
\hline $\begin{array}{l}\text { Mediana } \\
\text { Rango }\end{array}$ & 9 & 15 & $1,773.50$ & $-8.69^{* * *}$ \\
$\begin{array}{l}\text { Intercuartil } \\
\text { Rango de la }\end{array}$ & 6 & 6 & & \\
$\begin{array}{l}\text { Media } \\
n\end{array}$ & 70.23 & 143.43 & & \\
\hline
\end{tabular}

$* * * p<.001$

Los resultados del potencial intelectual, examinado a través del análisis descriptivo-comparativo, muestran que la mediana del grupo de los superdotados (Mediana $=26, I . R .=3$ ) fue significativamente mayor que la mediana del grupo no superdotado (Mediana $=18, I . R .=8), Z(210)=-10.34, p<.001$ (ver Cuadro 3).

También se observa que el grupo de los superdotados exhibe una distribución más homogénea de los puntajes que el grupo de los no superdotados, cuyo rango es también mayor que el anterior. Más aún, el análisis de la distribución de frecuencias exhibe una curva mesocúrtica, tendiente a la norma para el grupo no 
superdotado, mientras que para el grupo de los superdotados la curva tiende a ser leptocúrtica y sesgada negativamente.

\section{Cuadro 3}

Diferencias entre los puntajes del Test de Matrices Progresivas (CPM)

\begin{tabular}{lcccc}
\hline & No superdotados & Superdotados & $U$ & $Z$ \\
\hline $\begin{array}{l}\text { Mediana } \\
\text { Rango }\end{array}$ & 18 & 26 & 1,009 & $-10.34^{* * *}$ \\
Intercuartil & & & & \\
Rango de la & 8 & 3 & & \\
Media & 62.61 & 149.57 & & \\
$n$ & 105 & 107 & & \\
\hline
\end{tabular}

$* * * p<.001$

La relación entre la productividad intelectual, el desempeño creativo-figurativo y el potencial intelectual fue estudiada a través de los resultados del análisis discriminante, el cual revela que el Método del Portafolio, el TCT-DP y el CPM logran una correlación canónica de $r_{c} .90$ y un estadístico Wilks Lambda $l=$ .20 (ver Cuadro 4), que presenta un chi-cuadrado asociado de $X^{2}$ $(3, N=209)=335.26, p<.001$.

\section{Cuadro 4}

Análisis Wilks Lambda y Proporción F Univariada de las variables incluidas en el Análisis Discriminante

\begin{tabular}{lcc}
\hline \multicolumn{1}{c}{ Variable } & Wilks Lambda $(l)$ & $F$ \\
\hline Método del Portafolio & .49 & $214.01^{* * *}$ \\
TCT-DP & .75 & $70.39 * * *$ \\
CPM & .26 & $583.36 * * *$ \\
\hline
\end{tabular}

$n=209, g l=208, * * * p<.001$ 
Meta-análisis sobre estudios de identificación y atención al talento.

Estos resultados muestran que los perfiles de los superdotados y de los no superdotados son diferentes, como se muestra en la Figura 2.

$$
\begin{aligned}
& 1=\text { No Superdotados } \\
& 2=\text { Superdotados }
\end{aligned}
$$

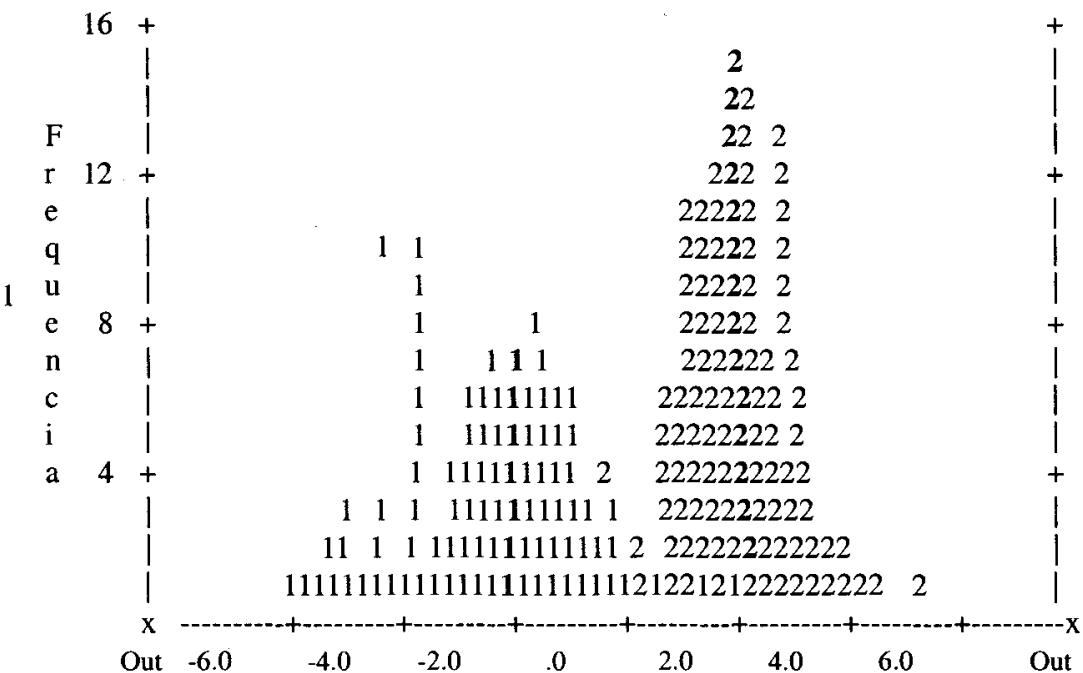

Class 1111111111111111111111111111111111122222222222222222222222222222222 Centroids

Figura 2. Distribución de los resultados de la función canónica discriminantes de los grupos estudiados $(n=209)$.

Los resultados del rendimiento escolar con la $U$ de Mann Whitney muestran que los superdotados (Mediana $=18, I . R .=$ 0.20 ) sistemáticamente logran puntajes más altos que los no superdotados (Mediana $=14, I . R .=3), z(215)=-12.15, p<.001$, como se observa en el Cuadro 5 . También se observa que los no superdotados presentan un elevado rango de puntajes, así como curva bimodal, mientras que el grupo de los superdotados exhibe una distribución homogénea a lo largo de 15 puntos y una curva leptocúrtica con sesgo negativo (ver Cuadro 5). 
Cuadro 5

Diferencias entre los puntajes de Rendimiento Escolar

\begin{tabular}{lcccc} 
& No superdotados & Superdotados & $U$ & $Z$ \\
\hline Mediana & 14 & 18 & 353.50 & $-12.15^{* * * *}$ \\
Rango Intercuartil & 3 & 0.20 & & \\
Rango de la Media & 58.71 & 160.70 & & \\
$n$ & 110 & 107 & & \\
\hline
\end{tabular}

$* * * p<.001$

La comparación entre la identificación del superdotado siguiendo la interrelación entre la productividad intelectual, el desempeño creativo-figurativo, el potencial intelectual y el rendimiento escolar se realizó utilizando los resultados de toda la correlación entre grupos de las variables discriminantes y la función canónica discriminante.

Los resultados revelan que los valores más altos fueron los de productividad intelectual, seguidos por rendimiento escolar y potencial intelectual. Los valores más bajos estuvieron relacionados al desempeño creativo-figurativo. Siguiendo el análisis de resultados, el perfil que nos permitirá discriminar al superdotado del no superdotado integrando las variables productividad intelectual, desempeño figurativo-creativo y capacidad intelectual potencial en la identificación del superdotado, comprende puntajes mayores a $30(\mathrm{Pc}>90)$ en el Método del Portafolio, puntajes mayores a $17(\mathrm{Pc}>90)$ en el $T C T-D P$ y puntajes mayores a $27(\mathrm{Pc}>95)$ en el $C P M$. Asimismo, el rendimiento escolar equivalente a la calificación A podría ser considerado un indicador significativo para la detección del superdotado en el aula de clases regular.

Los resultados del presente estudio revelan que existe una relación significativa entre la identificación del superdotado según el Modelo Multifactorial de Superdotación de Mönks (1992) y el rendimiento escolar en segundo grado en los colegios públicos de 
Meta-análisis sobre estudios de identificación y atención al talento.

Lima. Más aún, en caso de la necesidad de un proceso de detección rápido, el rendimiento escolar, expresado en las calificaciones obtenidas, podría ser utilizado como indicador pedagógico si y sólo si la calificación es realizada por profesores entrenados en los programas de articulación y en el segundo grado de los colegios públicos de Lima.

La discusión está organizada según (a) la caracterización de la productividad intelectual, el desempeño creativo-figurativo y el potencial intelectual de los participantes por separado, (b) la relación entre las tres variables estudiadas y (c) la comparación entre las tres variables según el rendimiento escolar.

\section{Caracterización de la productividad intelectual}

Los resultados concuerdan con los estudios realizados por Janos y Robinson (1985), quienes sugieren una clara relación entre la superdotación y factores asociados con la productividad intelectual, tales como: energía, vigor, entusiasmo, vitalidad, perseverancia, persistencia, ambición, competitividad, elevadas aspiraciones, curiosidad, exploración y situaciones de riesgo. También Hallahan y Kauffman (1978) con niños estadounidenses, Roe (1952) en su estudio sobre científicos eminentes y Feldman (1979) en su estudio sobre niños prodigio resaltan estas características como comunes a los talentosos. Más aún, Stanley (1984) señala la sed por el conocimiento como significativo en estos niños. McLeod y Cropley (1989) sugieren que este tipo de motivación y convicción aparece en personas con buena imagen de sí mismos y confiados en sus posibilidades de logro. En un intento por explicar la productividad intelectual a partir de la estimulación de adultos significativos en los superdotados de Hungría, Herskovitz y Gyarmathy (1995) asocian la productividad intelectual con el ambiente sociocultural que influye en el desarrollo de la superdotación. 
En relación al uso del Método del Portafolio como método de identificación, los resultados corroboran la propuesta de Grace (1997), así como la de Meisels y Steele (1991), quienes señalan la importancia del Método del Portafolio debido a que incorpora los procesos y características del desarrollo infantil en la evaluación del sistema escolar. Asimismo, apoya la propuesta de Calfee y Perfumo (1993), quienes en su análisis de las estrategias de evaluación en el sistema escolar, sugieren el uso de este método.

\section{Caracterización del desempeño creativo a través de producciones figurativas}

Los resultados concuerdan con los de Cropley (1994), Getzels (1987) y Urban $(1994,1995)$, quienes enfatizan el rol del desempeño creativo en la superdotación y concuerdan con los de Facaroaru (1985), quien en un estudio sobre los factores asociados al éxito en un grupo de ingenieros eminentes, mostró que algunas habilidades, tales como la generación de ideas novedosas, el reconocimiento de alternativas múltiples en la solución de un problema y la originalidad, entre otras asociadas con el desempeño creativo, son determinantes en el éxito profesional. En el Perú, los resultados concuerdan con los del CONCYTEC (1989) que considera que el desempeño creativo es un indicador de superdotación en los niños de Lima. Estos resultados concuerdan también con un estudio previo de Blumen (1996) realizado con niños superdotados de cinco años, que habitaban zonas rurales y que presentaron un desempeño significativamente mejor que los no superdotados. Se sugieren estudios futuros con el fin de explorar la naturaleza de las diferencias que pueden establecerse entre los superdotados y los no superdotados en el desempeño creativo diferente a la producción figurativa. 
Meta-análisis sobre estudios de identificación y atención al talento.

\section{Potencial intelectual}

La discrepancia significativa entre los puntajes de los superdotados y no superdotados a nivel de potencial intelectual concuerda con Humphreys (1985), quien señala al factor intelectual como determinante en la identificación del superdotado. También McLeod y Kluckmann (1985), Passow (1985), Yarborough y Johnson (1983) enfatizan la importancia de la inteligencia en la identificación del superdotado y su uso en el $80 \%$ de los programas de intervención en la mayoría de países. En el Perú, los pocos estudios que se refieren al proceso de identificación (CONCYTEC, 1989; González, 1991; Ruiz, 1991) lo consideran también como determinante. Tanto los estudios de González (1991) sobre adolescentes en Tacna, como los de Ruiz (1991) sobre rasgos de personalidad en mujeres adolescentes superdotadas, concuerdan con los hallazgos del presente estudio que consideran al factor intelectual como significativo en la identificación del superdotado, confirmando su importancia.

\section{Análisis de la combinación de los factores}

El análisis de la combinación de los factores revela la importancia de la motivación, creatividad y capacidad superior en la superdotación como constructo teórico, que se enmarca en la psicología del desarrollo. Cabe señalar que la triada personal del Modelo Multifactorial de Superdotación de Mönks requiere la presencia e interacción de los tres factores para que la capacidad potencial sea actualizada, lo cual le brinda al modelo una perspectiva multifactorial (McLeod \& Cropley, 1989). Asimismo, el rol fundamental de la capacitación docente, relevante en todo proceso de mejoramiento de la calidad educativa, es determinante en el proceso de identificación de los superdotados escolarizados. Estos resultados concuerdan con los de Pedulla, Airasian y Madaus (1980), quienes encuentran una correlación moderada 
entre la nominación de los profesores y los resultados de las pruebas psicométricas, señalando que los profesores tienden a identificar conductas asociadas con potencial académico tales como persistencia o rangos elevados de atención, que no son evaluadas en las pruebas tradicionales pese a ser importantes en la estimación del potencial académico.

En conclusión, el presente estudio nos brinda datos empíricos que permiten corroborar el uso del Modelo Multifactorial de Superdotación de Mönks para identificar a los niños superdotados de segundo grado de los colegios públicos de Lima, utilizando instrumentos estandarizados y contando con profesores calificados. Más aún, se corrobora que el uso de una combinación de medidas para la identificación del superdotado presenta mayores ventajas que el uso de una medida única en términos de confiabilidad y validez, tal como Hany (1993) sugirió en su estudio sobre dificultades metodológicas en la identificación del superdotado en Alemania. En este sentido, la probabilidad de cometer un error en el proceso de identificación del superdotado es mínima y apoya tanto el procedimiento como los resultados obtenidos. Asimismo, el currículo de base constructivista presenta elementos apropiados para la evaluación como parte de un proceso de detección que considere el rendimiento escolar. En este sentido, el proceso de identificación puede ser desarrollado en dos niveles: detección del desempeño académico e identificación siguiendo la interrelación de las variables estudiadas.

Se recomienda al sistema educativo peruano que todo proceso de identificación del superdotado se base en un modelo o teoría que le brinde sustento teórico y que conlleve a algún tipo de atención integral, pedagógica y/o psicológica. Las niñas y los niños no deberán formar parte de evaluaciones que no cuenten con bases científicas sólidas o con alternativas de derivación. En el caso del presente estudio, los participantes fueron incorporados en un estudio siguiente y complementario al proceso de identificación. Asimismo, es necesario establecer la metodología 
Meta-análisis sobre estudios de identificación y atención al talento.

que monitoree el proceso, dado que las decisiones diagnósticas deben ser realizadas en términos de resultados estadísticamente válidos, evitando decisiones subjetivas.

Finalmente, se sugiere una línea de investigación que corrobore los resultados obtenidos y que proponga nuevas estrategias de identificación, considerando la diversidad multicultural del Perú.

\section{Identificación del talento y la superdotación}

Un estudio reciente sobre los superdotados en desventaja cultural que además habitan en ambientes multiculturales del Perú (Blumen \& Cornejo, 2004) sugiere algunas formas para favorecer la validez y confiabilidad de las investigaciones. Primero, los resultados indican que el grado de acuerdo entre la identificación realizada por profesionales de la salud (pediatras, neurólogos y psiquiatras) en el curso de su trabajo, y el diagnóstico psicológico realizado sobre la base de una batería completa de evaluación, se encuentra en rangos de moderado (kappa $=0.57)$ a bueno (kappa $=0.85$ ). Sin embargo, el acuerdo entre la identificación realizada por profesores no capacitados o padres de familia y el criterio diagnóstico psicológico se encuentra en un rango de pobre (kappa $=0.24)$ a moderado (kappa $=0.53$ ) (Blumen \& Cornejo, 2004). Los resultados mejoran con profesores capacitados en la educación del talentoso. Es por ello necesario incorporar el tema de la superdotación y el talento, tanto a nivel de formación inicial como de formación continua en los docentes.

Segundo, parece que bajo ciertas circunstancias los psicólogos tienden a percibir a los superdotados como un grupo patológico. En algunos casos, la tendencia a patologizar a los adultos superdotados se ha debido a la inadecuación de algunas pruebas psicológicas populares (Blumen \& Cornejo, 2004).

Tercero, se han obtenido resultados importantes respecto al efecto del sesgo en el acuerdo entre el diagnóstico clínico y el de 
investigación, donde la diferencia de género no ha sido significativa. Sin embargo, sí se ha encontrado variación para los grupos étnicamente diferentes. En un estudio peruano centrado en la identificación de los superdotados en ambientes multiculturales, el acuerdo entre el diagnóstico clínico y el de investigación ha sido mejor para la muestra de la zona costera (mejor en áreas urbanas que en áreas rurales, $57 \%$ vs. $48 \%$ ), y peor para los niños de los Andes que para los de la amazonía (kappa $=0.43$ y 0.45 para los Andes; kappa $=0.55$ y 0.59 para la amazonía) (Blumen \& Cornejo, 2004). No es aún clara la razón por la cual los resultados en sesgo étnico varían a lo largo de los estudios. Sin embargo, podrían estar asociados a las diferencias multiculturales en la concepción de la superdotación (ver Cuadro 6).

\section{Cuadro 6}

Diferencias entre el paradigma occidental y algu nos factores asociados a la cosmovisión andina y a algunas etnias amazónicas en la concepción del talento (Blumen \& Cornejo, 2004)

\begin{tabular}{|c|l|l|l|}
\hline & Paradigma occidental & Cosmovisión andina $\left(^{*}\right)$ & $\begin{array}{l}\text { Cosmovisión de algunas } \\
\text { etnias amazónicas }\left(^{*}\right)\end{array}$ \\
\hline $\begin{array}{c}\text { Marco } \\
\text { teórico }\end{array}$ & $\begin{array}{l}\text { Teorías evolutivas y } \\
\text { del desarrollo. } \\
\text { Orientadas al proceso. }\end{array}$ & Divinidad, animismo, naturalismo. \\
\hline $\begin{array}{c}\text { El talento } \\
\text { como constructo }\end{array}$ & $\begin{array}{l}\text { Supone variables múltiples, } \\
\text { intrínsecas y extrínsecas al } \\
\text { ndividuo. }\end{array}$ & $\begin{array}{l}\text { Relacionadas al } \\
\text { colectivismo y } \\
\text { estrechamente vinculadas } \\
\text { a fenómenos naturales. }\end{array}$ & $\begin{array}{l}\text { Relacionadas con la } \\
\text { naturaleza, con } \\
\text { elementos de } \\
\text { colectivismo e } \\
\text { individualismo. }\end{array}$ \\
\hline $\begin{array}{c}\text { Identificación } \\
\text { del talentoso }\end{array}$ & $\begin{array}{l}\text { - En base al logro y } \\
\text { espećficas al dominio. } \\
\text { - Tendencia hacia la }\end{array}$ & $\begin{array}{l}\text { - Liderazgo a través del reconocimiento comunal. } \\
\text { - Conocimiento de temas asociados con la divinidad. } \\
\text { - Dominio de destrezas de supervivencia. } \\
\text { - Factores etnocéntricos y sociales. }\end{array}$ \\
\hline $\begin{array}{l}\text { Determinantes } \\
\text { en la expresión } \\
\text { del talento }\end{array}$ & Variables ambientales tales como la familia, la escuela, el trabajo y la comunidad. \\
\hline
\end{tabular}

(*) Muestra tomada en etnias que presentan contacto limitado con la cultura occidental. 
Meta-análisis sobre estudios de identificación y atención al talento.

Cuarto, cuando se refieren a talentosos latinoamericanos en desventaja cultural, es importante señalar que el nivel socioeconómico (NSE) está asociado a esta condición. Pese a que existen diferentes formas de medir el NSE, la mayoría incluye la cuantificación del ingreso familiar, la educación parental y el estatus ocupacional. Los estudios muestran que el NSE está asociado con un amplio rango de situaciones asociadas con la salud, la cognición y el desarrollo socio-emocional en los niños, cuyos efectos se observan en etapas previas al nacimiento y continúan a lo largo de su adultez, influyendo en el niño, en la familia y en la comunidad. Sus efectos serán moderados por las características propias de cada niño, así como por las características familiares y por los sistemas de apoyo externo de los cuales se puedan beneficiar (Blumen \& Cornejo, 2004).

\section{Capacitación docente en la educación del superdotado}

La formación inicial y la capacitación continua de los docentes deben incorporar temas asociados al talento y la superdotación, con el fin de brindar herramientas a los educadores para identificar a los superdotados y atender a sus necesidades en el aula de clases. Tomará muchos años el incluir a la niña y al niño superdotado en la formación del docente. Sin embargo, ellos están ya asistiendo a los colegios de toda América Latina. A lo largo de las últimas décadas se han desarrollado esfuerzos desde diferentes universidades tanto en Norteamérica como en Asia y Europa, con el fin de capacitar a los docentes en la educación del talentoso y del superdotado, cuyo impacto se observa, no sólo en la atención al superdotado y talentoso, sino en toda la población en general.

En el Perú este programa se inició en enero de 1999 y constituyó el primer curso del European Council for High Ability (ECHA) realizado en América Latina (Blumen, 2000, 2001). En

más de 500 horas, 21 profesores y psicólogos docentes de aulas 
integradas, se capacitaron y trabajan en la actualidad como agentes multiplicadores en las escuelas estatales de los diversos contextos multiculturales del Perú. El detalle de las investigaciones realizadas por tema de interés se puede ver en el Cuadro 7. Los egresados del Diploma ECHA, desarrollado en el Perú, asumieron el liderazgo del proyecto de integración en la escuela orientado hacia la detección de talentosos y/o superdotados en el aula de clases, desarrollados en siete departamentos del país. Ellos participan en el Plan Nacional de Atención a los Talentosos realizando programas de identificación, talleres de capacitación para los docentes en ejercicio y programas de enriquecimiento focalizados en la creatividad, la productividad y la emergencia de talentos múltiples.

\section{Cuadro 7}

Relación de temas desarrollados en el Diploma ECHA en el Perú (Blumen, 2000)

\begin{tabular}{|c|c|c|c|c|}
\hline $\begin{array}{l}\text { Area de } \\
\text { interés }\end{array}$ & Autor & Titulo & $\begin{array}{c}\text { Nivel de } \\
\text { investigación }\end{array}$ & Muestra \\
\hline & $\begin{array}{c}\text { Monroy y } \\
\text { Ruiz }\end{array}$ & $\begin{array}{l}\text { Programa de enriquecimiento en el } \\
\text { área lógico-matemático en el segundo } \\
\text { grado de educación primaria de un } \\
\text { centro estatal de la ciudad de Lima }\end{array}$ & Correlacional & $\begin{array}{l}60 \text { niños y niñas de } \\
\text { segundo grado de } \\
\text { colegio público en } \\
\text { Lima. }\end{array}$ \\
\hline \multirow[t]{4}{*}{$\begin{array}{l}\text { Lógico- } \\
\text { matemático }\end{array}$} & Martínez & $\begin{array}{c}\text { Programa de enriquecimiento lógico } \\
\text { matemático en niños de } 5 \text { años de } \\
\text { Huacho }\end{array}$ & $\begin{array}{l}\text { Descriptivo- } \\
\text { exploratorio }\end{array}$ & $\begin{array}{l}50 \text { niños y niñas de } 5 \\
\text { años de Huacho. }\end{array}$ \\
\hline & Panduro & $\begin{array}{c}\text { Enriquecimiento en lógico } \\
\text { matemática }\end{array}$ & $\begin{array}{l}\text { Descriptivo- } \\
\text { correlacional }\end{array}$ & $\begin{array}{l}47 \text { niños de quinto } \\
\text { grado de colegio } \\
\text { privado en Lima. }\end{array}$ \\
\hline & Costa & $\begin{array}{c}\text { Enriquecimiento para alumnas con } \\
\text { talento matemático en segundo de } \\
\text { secundaria en Piura }\end{array}$ & Correlacional & $\begin{array}{c}190 \text { alumnas de } \\
\text { segundo de secundaria } \\
\text { de colegio público } \\
\text { en Piura }\end{array}$ \\
\hline & Morales & $\begin{array}{l}\text { La comprensión lectora en adolescentes } \\
\text { talentosos que asisten a wi programa de } \\
\text { enriquecimiento en lectura con un } \\
\text { enforue metacognitivo y crítico }\end{array}$ & $\begin{array}{c}\text { Descriptivo } \\
\text { Estudio de casos }\end{array}$ & $\begin{array}{c}\text { Seis estudiantes de } \\
\text { alta capacidad intelec- } \\
\text { tual de } 11 \text { y } 16 \text { años } \\
\text { de Lima. }\end{array}$ \\
\hline
\end{tabular}


Meta-análisis sobre estudios de identificación y atención al talento.

\begin{tabular}{|c|c|c|c|c|}
\hline $\begin{array}{l}\text { Area de } \\
\text { interés }\end{array}$ & Autor & Título & $\begin{array}{c}\text { Nivel de } \\
\text { investigación }\end{array}$ & Muestra \\
\hline \multirow{4}{*}{$\begin{array}{r}\text { Verbal/ } \\
\text { literaria }\end{array}$} & Cajahuaringa & $\begin{array}{c}\text { El programa PRECRELI y la } \\
\text { creación de textos literarios en niños } \\
\text { de cuarto grado de educación } \\
\text { primaria }\end{array}$ & $\begin{array}{l}\text { Descriptivo- } \\
\text { exploratorio }\end{array}$ & $\begin{array}{l}40 \text { niñas y niños de } \\
\text { cuarto grado de } \\
\text { colegio público en } \\
\text { Lima. }\end{array}$ \\
\hline & García & $\begin{array}{c}\text { Enriquecimiento en la comprensión } \\
\text { lectora }\end{array}$ & Correlacional & $\begin{array}{c}56 \text { alumnas de cuarto } \\
\text { grado de Ica. }\end{array}$ \\
\hline & Alvarez & $\begin{array}{l}\text { Programa experimental de enrique- } \\
\text { cimiento en técnicas básicas de } \\
\text { elaboración de mapas conceptuales } \\
\text { en niños y niñas de tercer grado de } \\
\text { una escuela estatal de La Victoria }\end{array}$ & Exploratorio & $\begin{array}{c}52 \text { alumnos de } 8 \text { a } 11 \\
\text { años de La Victoria, } \\
\text { Lima. }\end{array}$ \\
\hline & Castro & $\begin{array}{c}\text { Programa de enriquecimiento de la } \\
\text { creatividad literaria en niños de } \\
\text { educación primaria }\end{array}$ & $\begin{array}{l}\text { Descriptivo- } \\
\text { correlacional }\end{array}$ & $\begin{array}{c}50 \text { alumnos de } 9 \text { a } 11 \\
\text { años de colegio } \\
\text { público en Lima. }\end{array}$ \\
\hline Psicomotriz & Cornejo & $\begin{array}{c}\text { Innovación estratégica y desarrollo } \\
\text { psicomotriz del niño en educación } \\
\text { primaria }\end{array}$ & $\begin{array}{l}\text { Descriptivo- } \\
\text { correlacional }\end{array}$ & $\begin{array}{c}53 \text { alumnos de quinto } \\
\text { grado, turno vespertino } \\
\text { en Lima }\end{array}$ \\
\hline \multirow{3}{*}{ Preescolar } & $\begin{array}{l}\text { Levy y } \\
\text { Mansilla }\end{array}$ & $\begin{array}{c}\text { Programa de enriquecimiento en el } \\
\text { área verbal para niños de } 5 \text { años de } \\
\text { edad }\end{array}$ & $\begin{array}{l}\text { Descriptivo- } \\
\text { correlacional }\end{array}$ & $\begin{array}{l}58 \text { niños de Kinder, } \\
\text { entre } 5.1 \text { y } 6.4 \text { años, } \\
\text { colegio público y } \\
\text { privado en Lima. }\end{array}$ \\
\hline & Valdivia & $\begin{array}{l}\text { Programa de enriquecimiento } \\
\text { creativo figurativo de niños de } 5 \\
\text { años }\end{array}$ & $\begin{array}{l}\text { Descriptivo- } \\
\text { correlacional }\end{array}$ & $\begin{array}{c}50 \text { niños entre } 5.2 \text { y } \\
5.9 \text { años, de colegios } \\
\text { públicos en Lima. }\end{array}$ \\
\hline & $\begin{array}{l}\text { García y } \\
\text { Paredes }\end{array}$ & $\begin{array}{c}\text { Prograna Jugando Aprendo y el } \\
\text { desarrollo del pensamiento divergen } \\
\text { te en niños talentosos }\end{array}$ & Descriptivo & $\begin{array}{c}10 \text { niñas y niños de } \\
\text { alta capacidad intelec- } \\
\text { tual, entre } 5.4 \text { y } 6 \text { años } \\
\text { en Lima. }\end{array}$ \\
\hline \multirow{2}{*}{ Padres } & Gutiérrez & $\begin{array}{c}\text { Programa de enriquerimiento } \\
\text { Despliegue, habilidades cognitivas } \\
\text { y hábitos de estudio para niños de } \\
\text { quinto grado }\end{array}$ & Experimental & $\begin{array}{c}63 \text { estudiantes de } \\
\text { quinto grado de colegio } \\
\text { privado parroquial en } \\
\text { Lima. }\end{array}$ \\
\hline & Melgar & $\begin{array}{c}\text { Indicadores de superdotación y } \\
\text { talento según padres, profesores y } \\
\text { pares }\end{array}$ & Descriptivo & $\begin{array}{c}126 \text { padres, } 71 \\
\text { profesores y } 470 \text { pares. }\end{array}$ \\
\hline $\begin{array}{l}\text { Capacitación } \\
\text { docente }\end{array}$ & Blumen & $\begin{array}{c}\text { Atención integral para el talentoso } \\
\text { en el aula de clases }\end{array}$ & Experimental & $\begin{array}{l}217 \text { estudiantes de } \\
\text { segundo grado. }\end{array}$ \\
\hline
\end{tabular}


Es necesario evaluar alternativas administrativas que cubran las necesidades de los niños con talento y superdotación en las escuelas públicas, diversificando la atención hacia los talentos múltiples según las características multiculturales y plurilingües del Perú. Es esencial subrayar que el apoyo emocional y pedagógico para las niñas y los niños talentosos facilita el desarrollo de sus talentos, especialmente para aquellos que viven en condición de desventaja cultural y pobreza, que constituyen la gran mayoría de la población escolar en el Perú y en América Latina.

\section{Efectos de un programa de capacitación docente en la creatividad, cognición y rendimiento escolar}

Se elaboró un programa de capacitación docente con el fin de estudiar el enriquecimiento cognitivo en el aula de clases en niñas y niños con talento académico. La pregunta que se respondió fue la siguiente: ¿es el programa de capacitación docente efectivo en mejorar el desempeño creativo-figurativo, el desarrollo cognitivo y el rendimiento académico en niñas y niños de segundo grado de colegios públicos de Lima?

Las variables independientes (VI) fueron:

- Programa de capacitación docente: variable experimental que consiste en la participación en el programa de capacitación.

- Nivel de capacidad: variable atributiva relacionada con el nivel de capacidad de los participantes, detectada utilizando un programa de detección en un estudio anterior (Blumen, 1998).

Las variables dependientes (VD) fueron:

- Desempeño creativo-figurativo, definido y medido según el TCT-DP de Urban y Jellen (1986). 
Meta-análisis sobre estudios de identificación y atención al talento.

- Desarrollo cognitivo, definido y medido a través del Cog At Forma 5 de Thorndike y Hagen (1993).

- Rendimiento escolar, definido y medido según los resultados generales del primer y segundo semestre del año académico.

Las variables de control fueron el grado, las calificaciones del docente, la edad, el tipo de colegio y el suplemento nutricional complementario (todos los participantes recibieron una comida extra mientras estaban en el colegio).

Es un estudio de campo de tipo experimental que pretende llegar a un nivel explicativo. Se utilizó el diseño de Medidas Repetidas, con Tiempo de Administración (pre- y post-test) como factor intra-sujeto de 2 niveles, aplicado a las variables dependientes (desempeño creativo-figurativo, desarrollo cognitivo y rendimiento escolar). Como factores intra-sujetos de dos niveles se utilizó el nivel de capacidad (talentoso vs. no talentoso) y la Condición (experimental y control).

Los participantes fueron 231 estudiantes de segundo grado de los colegios públicos de Lima, 125 talentosos (64 varones y 61 niñas) y 106 no talentosos (57 varones y 49 niñas), con un rango de edad de 6 a 8 años, Media $=7$ años 4 meses, $D S=9.47$ meses y Mediana $=7$ años 3meses. De ellos, 69 talentosos (34 varones y 35 niñas) fueron asignados aleatoriamente al Grupo Experimental (GE) y 56 (30 varones y 26 niñas) al Grupo Control (GC). También 61 no talentosos (35 varones y 26 niñas) fueron asignados al GE y 45 (22 varones y 23 niñas) al GC. Todos los participantes completaron los formularios sobre el consentimiento informado en el programa de intervención propuesto.

Los participantes del GC fueron apareados con el GE por edad, género, colegio, grado y rendimiento académico. Se aplicaron $t$-tests de dos colas en la variable edad $t(229)=-.08, p$ $=.937$, con el fin de establecer si existía alguna diferencia en las medias. Se utilizó el estadístico chi-cuadrado para evaluar las diferencias de género. No se encontraron diferencias significativas 
entre los grupos experimental y control $X^{2}(1, N=231)=0.13, p$ $=.720$.

\section{Programa de capacitación docente}

El principal objetivo de este programa fue entrenar a profesores en el diseño e implementación de actividades para niños que exhibían un desempeño bajo o muy superior en el aula de clases, siguiendo el modelo procedimental de cambio conductual de Avalos (1993), que concibe a la capacitación docente como un proceso circular que supone cambios en el marco interpretativo del entrenado. Los participantes fueron 45 profesoras de segundo grado de primaria de 21 colegios públicos de la ciudad de Lima, cuyas edades estaban en un rango de 27 a 49 años (Media $=35 ; D S=5.10 ;$ Moda $=38$ ). Luego de inscribirse, los profesores tomaron el test TCT-DP y el cuestionario sobre educación del talentoso antes de iniciar los talleres del programa. Los resultados del taller revelan mejoras significativas en el desempeño creativo-figurativo, $t$-test apareado $(47)=-12.75, p<.001$, siendo los puntajes post-test $(M=39.44$; $D S=7.89)$ mejores que los pretest $(M=31.42, D S=7.47)$. El puntaje de logro en el conocimiento adquirido en la educación del superdotado también exhibió una mejora significativa, $t$-test apareado $t(47)=-11.82, p<.001$, siendo los puntajes posttest $(M=15.33, D S=0.98)$ mejores que los pretest $(M=13.15, D S=$ 1.52). Las actividades de monitoreo continuaron a lo largo de intervalos de tres meses.

\section{Programa de intervención}

El programa de intervención consistió en ser expuesto a lecciones cuatro veces por semana, a lo largo de 10 semanas, con 
Meta-análisis sobre estudios de identificación y atención al talento.

su monitoreo correspondiente. Cada tratamiento experimental semanal consistía en una sesión interactiva de una hora. Los procedimientos de intervención fueron estandarizados y estrictamente monitoreados por el investigador y los profesores capacitados, quienes fueron los administradores del programa de intervención. La intervención contempló el agrupamiento homogéneo y seis lecciones educativas en las siguientes áreas: comprensión, memoria, evaluación, solución de problemas convergentes, solución de problemas divergentes y manejo de tiempo y estrés. El diseño de la atmósfera del aula consideró la propuesta de De Corte (1994) sobre el contexto apropiado para la actividad de aprendizaje, en términos de una concepción constructivista moderada de aprendizaje.

El análisis de resultados contempló numerosas ANOVAs de Medidas Repetidas con análisis intra-sujetos, teniendo a la Capacidad (talentoso vs. no talentoso) y la Condición (control vs. experimental) como factores inter-sujeto, y al Tiempo de Administración (pre y post-test) como factor intra-sujeto, con el fin de evaluar las diferencias en el desempeño creativo-figurativo, las habilidades cognitivas y el rendimiento académico de los participantes.

Los resultados del $T C T-D P$ muestran que hubo un efecto principal para Capacidad $F(1,227)=123.90, p<.001$, con los talentosos exhibiendo un mejor desempeño $(M=16.70, D E=$ 4.56) que los no talentosos $(M=10.01, D E=0.42)$, y para la Condición $F(1,227)=12.45, p<.01$, con el grupo experimental exhibiendo un mejor desempeño $(M=14.50, D E=6.08)$ que el grupo control $(M=12.51, D E=4.98)$. No se encontraron efectos de la interacción Capacidad x Condición $F(1,227)=0.13, p=$ .718. Los Cuadros 8 y 9 ayudan a interpretar los resultados generales de este estudio. 
Sheyla Blumen de Pardo

\section{Cuadro 8}

Análisis de Varianza para Medidas Repetidas (Pre y Post-test) del TCT-DP, CogAt y Rendimiento Escolar por Tipo y Grupo

\begin{tabular}{|c|c|c|c|c|}
\hline \multirow[b]{2}{*}{ Fuente } & \multicolumn{4}{|c|}{$F$} \\
\hline & $\overline{d f}$ & $\mathrm{CP}$ & $\mathrm{CA}$ & $\overline{\mathrm{SA}}$ \\
\hline & & Inter-sujetos & & \\
\hline Capacidad (A) & 1 & $123.90 * * *$ & $15.78 * * *$ & $424.75 * * *$ \\
\hline Condición (C) & 1 & $12.45 * *$ & $25.35 * * *$ & $16.72 * * *$ \\
\hline$A \times C$ & 1 & 0.13 & 0.59 & 0.71 \\
\hline \multirow[t]{2}{*}{ Error } & 227 & $(40.95)$ & $(460.51)$ & (3.98) \\
\hline & & Intra-sujetos & & \\
\hline Tiempo (T) & 1 & 2.73 & $98.05 * * *$ & $20.85 * * *$ \\
\hline $\mathrm{T} \times \mathrm{A}$ & 1 & $12.71 * * *$ & 2.95 & $54.32 * * *$ \\
\hline $\mathrm{T} \times \mathrm{C}$ & 1 & $26.16 * * *$ & 0.002 & $62.89 * * *$ \\
\hline $\mathrm{T} \times \mathrm{A} \times \mathrm{C}$ & $i$ & 1.24 & 0.033 & 2.20 \\
\hline Error $(\mathrm{T})$ & 227 & (15.13) & $(78.697)$ & $(0.31)$ \\
\hline
\end{tabular}

Nota: Los valores en paréntesis representan la media al cuadrado de los errores. $\mathrm{CP}=$ Desempeño Creativo-Figurativo; $\mathrm{CA}=$ Habilidades Cognitivas; $\mathrm{SA}=$ Rendimiento Escolar.

${ }^{*} p<.05 ;{ }^{* *} p<.01 ;{ }^{* * *} p<.001$.

\section{Cuadro 9}

TCT-DP, CogAt y Rendimiento Escolar para el Pre, Post y la Media Pre-Post, como una función de la Capacidad y de la Condición

\begin{tabular}{|c|c|c|c|c|c|}
\hline & & \multicolumn{2}{|c|}{ Capacidad } & \multicolumn{2}{|c|}{ Condición } \\
\hline & & $\begin{array}{c}\text { No-T } \\
(n=106)\end{array}$ & $\begin{array}{c}\mathrm{T} \\
(n=125)\end{array}$ & $\begin{array}{l}\text { Control } \\
(n=101)\end{array}$ & $\begin{array}{l}\text { Experimental } \\
\qquad(n=130)\end{array}$ \\
\hline & Pre & & & & \\
\hline & $M$ & 8.94 & 16.93 & 13.24 & 13.28 \\
\hline & $D E$ & 5.14 & 3.85 & 5.58 & 6.32 \\
\hline \multirow[t]{7}{*}{$T C T-D P$} & Post & & & & \\
\hline & $M$ & 11.08 & 16.46 & 11.78 & 15.71 \\
\hline & $D E$ & 5.78 & 6.70 & 5.91 & 7.03 \\
\hline & Media & & & & \\
\hline & Pre-Post & & & & \\
\hline & $M$ & 10.01 & 16.70 & 12.51 & 14.50 \\
\hline & $D E$ & 0.42 & 4.56 & 4.98 & 6.08 \\
\hline
\end{tabular}


Meta-análisis sobre estudios de identificación y atención al talento.

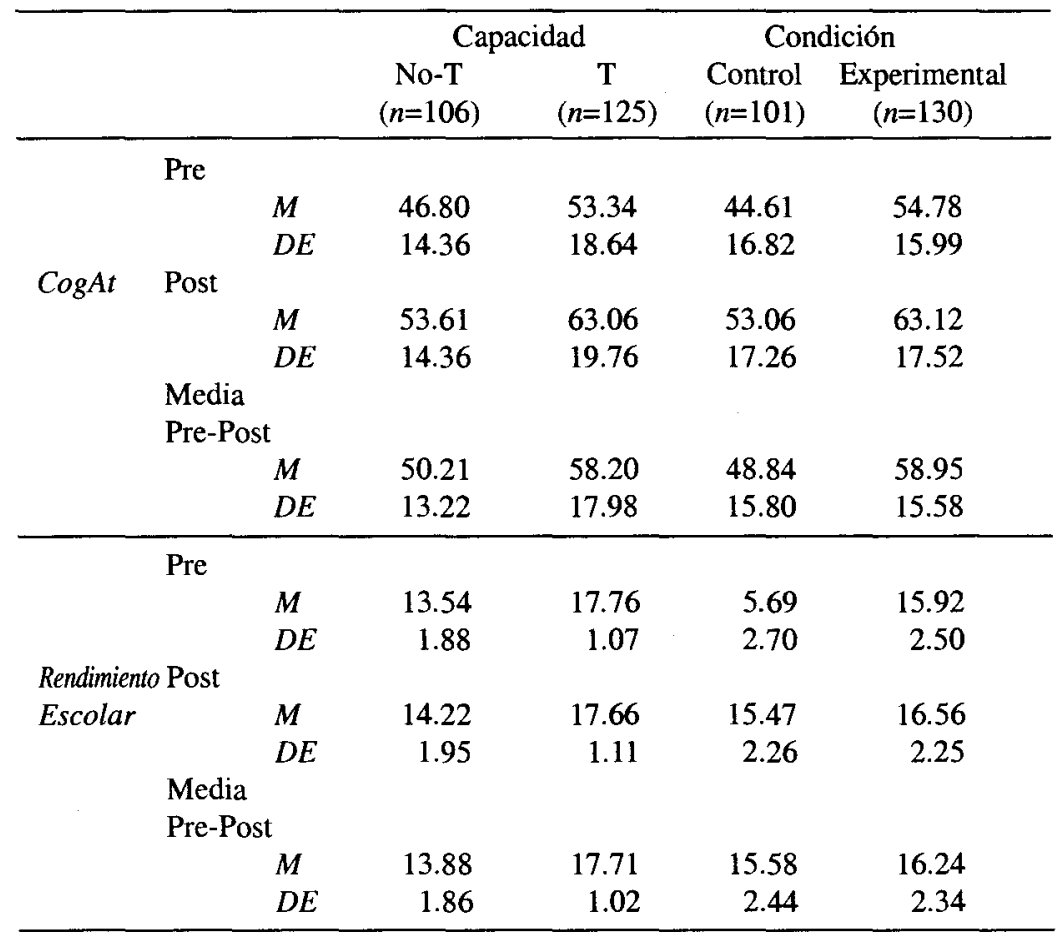

No se encontraron resultados significativos para el Tiempo $F(1,227)=2.73, p=.100$. Sin embargo, se observó una interacción Capacidad x Tiempo significativa $F(1,227)=12.71, p$ $<.001$ en el desempeño creativo-figurativo, donde los puntajes de los no talentosos declinaron durante el tiempo $\left(M_{\mathrm{pre}}=8.94, D E=\right.$ 5.14 y $\left.M_{\text {post }}=11.8, D E=5.78\right)$, mientras que en los talentosos se observó una baja leve $\left(M_{\text {pre }}=16.93, D E=3.85\right.$ y $M_{\text {post }}=16.46$, $D E=6.70)$.

El entrenamiento también afectó el desempeño creativofigurativo de los participantes, observándose un efecto de interacción Condición x Tiempo significativa $F(1,227)=26.16, p$ $<.001$, con el grupo experimental mejorando $\left(M_{\text {pre }}=13.28, D E=\right.$ 
6.32 y $M_{\text {post }}=15.71, D E=7.03$ ), mientras que el grupo control no lo hace $\left(M_{\text {pre }}=13.24, D E=5.58\right.$ y $\left.M_{\text {post }}=11.78, D E=5.91\right)$.

Los resultados del Cog At mostraron un efecto principal para la Capacidad $F(1,227)=15.78, p<.001$, con el grupo talentoso exhibiendo un mejor funcionamiento cognitivo $(M=58.20, D E=$ 17.98) que el no talentoso $(M=50.21, D E=13.22)$ y para la Condición $F(1,227)=25.35, p<.001$, con el grupo experimental exhibiendo un mejor desempeño $(M=58.95, D E=15.58)$ que el grupo control $(M=48.84, D E=15.80)$. No hubo efecto de interacción Capacidad x Condición. Asimismo, se observó un efecto principal por Tiempo $F(1,227)=98.05, \mathrm{p}<.001$, con el grupo post-test exhibiendo un mejor funcionamiento $(M=58.72$, $D E=18.08)$ que el pretest $(M=50.34, D E=17.09)$.

$\mathrm{El}$ análisis de rendimiento académico mostró que hay un efecto principal para Capacidad $F(1,227)=424.75, p<.001$, con los talentosos exhibiendo un mejor desempeño $(M=17.71, D E=$ 1.02) que los no talentosos $(M=13.88, D E=1.86)$, y para la Condición $F(1,227)=16.72, p<.001$, con el grupo experimental exhibiendo un mejor desempeño $(M=16.24, D E=2.34)$ que el grupo control $(M=15.58, D E=2.44)$.

Se observaron efectos principales por Tiempo $F(1,227)=$ $20.85, p<.001$, con el grupo post-test exhibiendo un mejor funcionamiento $(M=16.08, D E=2.31)$ que el pretest $(M=$ $15.82, D E=2.58)$. Se determinó un efecto de interacción Capacidad $\mathrm{x}$ Tiempo $F(1,227)=54.32, p<.001$, en relación al rendimiento académico, donde los no talentosos mejoraron $\left(M_{\text {pre }}=\right.$ $13.54, D E=1.88$, and $\left.M_{\text {post }}=14.22, D E=1.95\right)$, mientras que en los talentosos se observó un ligero decremento $\left(M_{\mathrm{pre}}=17.76, D E\right.$ $=1.07$, y $\left.M_{\text {post }}=17.66, D E=1.11\right)$. El entrenamiento afectó también el desempeño académico de los participantes. Se observó un efecto de interacción significativo $F(1,227)=62.89, p<.001$, con mejora en el grupo experimental $\left(M_{\text {pre }}=15.92, D E=2.50\right.$, y $\left.M_{\text {post }}=16.56, D E=2.25\right)$ mientras que el grupo control disminuyó $\left(M_{\text {pre }}^{\text {post }}=15.69, D E=2.70\right.$, y $\left.M_{\text {post }}=15.47, D E=2.26\right)$. 
Meta-análisis sobre estudios de identificación y atención al talento.

Los resultados revelan que hay efectos significativos del tratamiento experimental en el desempeño creativo-figurativo y en el rendimiento académico, siempre y cuando se cuente con la presencia de profesores capacitados y entrenados. Más aún, el tratamiento muestra una influencia positiva tanto para los talentosos como para los no talentosos.

\section{Efectos en el desempeño creativo-figurativo}

Los resultados muestran que si el talentoso se desarrolla en un ambiente estimulante, las oportunidades para lograr su potencial se incrementarán. Asimismo, los estudiantes no talentosos también tienden a producir en niveles académicamente elevados cuando son ubicados en grupos de su misma capacidad, sin embargo, las ganancias no son tan significativas para los talentosos. Nuestros resultados apoyan la comprensión científica del contexto facilitador, así como sus componentes principales (Mooney, 1963): una atmósfera adecuada y el agente facilitador (profesor calificado y entrenado en el desempeño creativo). Más aún, pese a que la creatividad como constructo es difícil de definir, es posible desarrollar desempeños creativos en sistemas educativos que los promuevan.

\section{Efectos en el funcionamiento cognitivo}

El hecho de que el programa no tenga un efecto significativo en el funcionamiento cognitivo puede ser interpretado en términos de que el efecto fue demasiado suave como para ser detectado. Más aún, cabe señalar que los atributos personales y las destrezas de comprensión, memoria, evaluación, solución de problemas convergentes y divergentes, y manejo de estrés requieren un periodo de tiempo mayor para ser desarrolladas (Silverman, 1993). 
Sheyla Blumen de Pardo

\section{Efectos en el desempeño académico}

Los niños talentosos tienden a tener mejor desempeño académico dado que son individuos competitivos que experimentan mayores situaciones de éxito a lo largo de su vida (Sternberg, 1996). El efecto de la intervención se puede explicar desde la perspectiva del logro en relación a las teorías conductuales, así como en función a la práctica en la activación de áreas asociadas a la acción y al logro. En esta medida, los resultados concuerdan con Lohman (1992), quien señala que el desempeño académico es una medida significativa del producto de la experiencia.

Los resultados sugieren que es posible atender a las necesidades de los niños talentosos dentro del aula regular si contamos con profesores entrenados para ello. En este sentido, queda aún la necesidad de establecer programas de capacitación constante para profesores, especialmente en los países en desarrollo. Es de suma importancia resaltar que en contextos de desventaja cultural y multiculturalidad en países en desarrollo, la escolaridad representa la única oportunidad que tienen los niños para adquirir algunas de las destrezas necesarias para tener éxito en la sociedad. Esto es especialmente relevante en un mundo cambiante en donde la estabilidad económica y política de los países depende de las capacidades de sus ciudadanos para adaptarse a los cambios.

\section{Discusión}

Numerosos psicólogos y educadores están poco familiarizados con la literatura científica respecto a las intervenciones terapéuticas y pedagógicas. Esta es una de las razones por las cuales las decisiones de tratamiento son en ocasiones inapropiadas. El problema de las intervenciones inapropiadas es en parte un tema 
Meta-análisis sobre estudios de identificación y atención al talento.

de evaluación y no sólo abarca la situación de intervención. Al parecer, algunos psicólogos y educadores toman decisiones inapropiadas al asesorar a los padres o profesores acerca de las necesidades de su niño talentoso, así como al formular los planes de intervención.

Diversos estudios en países desarrollados (Colangelo, Assouline \& Gross, 2004), así como en países en desarrollo (Alencar et al., 2000; Blumen, 2003), establecen formas efectivas para brindar los retos necesarios para el desarrollo de los talentosos y/o superdotados. Sin embargo, los profesionales de la salud, los psicólogos y educadores no tienen aún el éxito esperado en el desarrollo de procedimientos administrativos para llevarlo a la práctica. La principal barrera la constituyen los mitos y las concepciones populares erróneas respecto a los efectos negativos tanto a nivel emocional como social de los programas de intervención, pese a que éstas no cuentan con ningún soporte científico que los fundamente, más allá que las creencias populares (Colangelo et al., 2004).

Más aún, algunos programas de entrenamiento en la educación del talentoso pueden orientar hacia decisiones poco sólidas, al ofrecer créditos por la educación continua en una variedad de técnicas pedagógicas que no presentan soporte empírico (Blumen, 2001). Uno de los resultados más importantes en la toma de decisiones tiene que ver con el sesgo étnico. Se han observado sesgos étnicos en las recomendaciones hacia los programas de enriquecimiento en algunos países de Latinoamérica (Alencar et al., 2000). Estos hallazgos fueron replicados en un estudio entre niños talentosos de zonas urbanas y rurales del Perú, presentando resultados similares (Blumen et al., 2004). Existe una tendencia bastante generalizada a sugerir programas de enriquecimiento verbal o de enriquecimiento a través de las artes visuales para los niños talentosos en desventaja cultural que habitan zonas rurales. Mientras que se sugieren programas de enriquecimiento en matemáticas o ciencias para los talentosos en desventaja cultural 
que habitan en zonas urbanas, posiblemente debido a las condiciones de acceso a los materiales y a los docentes de mayor nivel de capacitación en estas áreas.

Finalmente, nos queda aún un tema sin resolver y está relacionado con la naturaleza del talento. Si el talento es considerado como una capacidad íntimamente asociada al estar informado acerca de las respuestas correctas frente a las preguntas, entonces el talento en los países de América Latina estará estrechamente vinculado con las oportunidades de acceso hacia una educación de calidad, lo cual escasea entre la mayoría de niños que habitan bajo condición de pobreza. Sin embargo, si el talento es considerado como la capacidad de realizar las preguntas adecuadas en las condiciones pertinentes, entonces las escuelas se convertirán en un espacio para el aprendizaje inquisitivo, marcando distancia con el estatus socioeconómico y dependerán de profesores capacitados y padres preocupados. Tal como sostenía Brockman (2002), en el futuro de la educación, la inteligencia no será asociada más con la capacidad de estar informado, sino con la capacidad de saber qué preguntas realizar. Por lo tanto, lo que será valorado serán las preguntas adecuadas y pertinentes a cada situación.

La presente revisión nos muestra que la identificación del talentoso en desventaja cultural está en el medio de un cambio altamente significativo. Nuevas presiones están forzando a los psicólogos y educadores a demostrar que sus técnicas de evaluación son adecuadas en términos costo-beneficio, presentan fundamentos científicos y son culturalmente sensibles. En este sentido, se ofrecen las siguientes conclusiones:

- Las limitaciones económicas en los servicios de salud, psicología y educación, que caracteriza a los países de América Latina no desaparecerán en tiempos próximos. Por lo tanto, los psicólogos y educadores deberán encontrar técnicas de identificación que provean información válida y 
Meta-análisis sobre estudios de identificación y atención al talento.

confiable. Es probable que algunas de éstas serán abandonadas y emergerán nuevas técnicas e instrumentos.

- En la medida que la población mundial se vuelva más diversa, la evaluación multicultural se volverá una condición importante. Por muchos años, los temas multiculturales fueron dejados de lado, considerándose periféricos a la investigación y la práctica tanto en la evaluación clínica como en la educacional. En el futuro, tendrán un rol preponderante en toda situación de evaluación como una necesidad para la mejor comprensión de las personas que habitan nuestro planeta.

- El desarrollo de la ciencia introducirá formas y elementos inesperados en el proceso de identificación. La investigación en la próxima década ayudará a explicar cómo opera la diversidad a partir múltiples mecanismos que afectan de manera simultánea el curso del desarrollo, cómo varía el desarrollo a través de los grupos étnicos y culturales y cómo los diferentes componentes de las variables socioeconómicas afectan los diferentes sistemas del desarrollo.

\section{Referencias}

Alencar, E., Blumen-Pardo, S. \& Castellanos-Simons, D. (2000). Programs and practices for identifying and nurturing giftedness and talent in Latin American countries. En K. A. Heller, F. J. Mönks, R. J. Sternberg \& R. F. Subotnik (Eds.), International handbook of giftedness and talent (2da. Ed.) (pp. 817-828). Oxford: Elsevier Science.

Alencar, E., De Souza, D. \& Blumen-Pardo, S. (2002). Trends in gifted education in South America: The Brazilian and 
Peruvian scenario. Gifted and Talented International, 17(1), 7-12.

Ardila, A., Rosselli, M. \& Puente, A. E. (1994). Neuropsychological evaluation of the Spanish speaker. Nueva York: Plenum.

Avalos, B. (1993). Teacher training in developing countries: Lessons from research. En J. P. Farrell \& J. B. Oliveira (Eds.), Teachers in developing countries: Improving effectiveness and managing costs (pp. 175-186). Washington, DC: EDI Seminar Series.

Ayala, A. \& Farfan, W. (1997, octubre). Efectos de un programa psicopedagógico para el mejoramiento de la comprensión lectora. Documento presentado en el VIII Congreso Nacional de Psicología, Lima, Perú.

Bechervaise, N. E. (1996). Gifted education in a multicultural Australia: Gifted programs as a divisive element in the educational aspiration of migrant population. Comunicación presentada en la Conferencia Internacional Anual en Investigación Educacional de la Australian Association of Research in Education (AARE), Universidad de Sydney, Australia.

Blumen, S. (1996, octubre). Nurturing and promotion of giftedness and creativity in early childhood in Peru. Documento presentado en el VIII Congreso del European Council for High Ability, Viena, Austria.

Blumen, S. (1998). Factores asociados a la identificación del talentoso en Lima. Lima: PUCP.

Blumen, S. (2000). Identification of and attention for the highly able in Lima. Tesis doctoral no publicada, Universidad de Nimega, Holanda.

Blumen, S. (2001) (Ed.). Enriquecer el talento en el aula de clases. Lima: Ministerio de Educación/Programa de Mejoramiento de la Calidad Educativa-MECEP.

Blumen, S. (2002). Effects of a teacher training workshop on creativity, cognition and school achievement in gifted and 
Meta-análisis sobre estudios de identificación y atención al talento.

non-gifted second grade students in Lima, Peru. High Ability Studies, 13(1), 47-58.

Blumen, S. (2003, julio). State-of-art in the identification of the academically gifted in Latin America. Simposio presentado en el XXIX Congreso Interamericano de Psicología, Lima, Perú.

Blumen, S. (2004, setiembre). Diversity as a milestone in the identification of the culturally disadvantage highly able. Documento presentado en la IX Conferencia del European Council for High Ability, Pamplona, España.

Blumen, S. \& Cornejo, M. (2004, noviembre). Efectos del origen étnico y varianza en la información, en la identificación del superdotado según los servicios de salud, educación y el diagnóstico psicológico. Documento presentado en el $\mathrm{V}$ Congreso Iberoamericano de Superdotación y Talento, Loja, Ecuador.

Brockman, J. (2002). The next fifty years. Science in the first half of the twenty-first century. Nueva York: Vintage Books.

Calfee, R. C. \& Perfumo, P. (1993). The student portfolios: Opportunities for a revolution in assessment. Journal of Reading, 36(7), 532-537.

Colangelo, N., Assouline, S. G \& Gross, M. (2004). A nation deceived: How schools hold back America's brightest students. Washington: Templeton National Report on Acceleration.

CONCYTEC (1989). Programa de detección y apoyo a niños superdotados. Lima: Consejo Nacional de Ciencia y Tecnología - CONCYTEC.

Corbalán, J., Martínez, F. \& Donolo, D. (2003). CREA. Inteligencia creativa. Una medida cognitiva de la creatividad. Madrid: TEA Ediciones.

Cropley, A. J. (1994). Creative intelligence: A concept of "true" giftedness. European Journal for High Ability, 5, 6-23. 
Cuellar, I. (2000). Acculturation as a moderator of personality and psychological assessment. En R. H. Dana (Ed.), Handbook of Cross-Cultural and Multicultural Personality Assessment (pp. 113-129). Nueva Jersey: Erlabaum.

De Corte, E. (1994, octubre). Learning and high ability: A perspective from research in instructional psychology. Documento presentado en la IV Conferencia del European Council for High Ability (ECHA), Nimega, Holanda.

Esquivel, G. (2000). Creativity and giftedness in culturally diverse students. Perspectives on creativity. Nueva Jersey: Hampton Press.

Facaroaru, C. (1985). Kreativitat e Wissenschaft und Technik. Berna: Huber.

Feldman, D. H. (1979). The mysterious case of extreme giftedness. En A. H. Passow (Ed.), The gifted and the talented: Their education and development. Chicago, IL: NSSE Yearbook.

Getzels, J. (1987). Creativity, intelligence, problem findings: Retrospect and prospect. En S. Isaksen (Ed.), Frontiers of creativity research (pp. 88-102). Buffalo, Nueva York: Bearly. González, A. (1991). Detección de talentos y desarrollo para la regionalización. Lima: CONCYTEC.

Grace, C. (1997). The portfolio and its gift: Developmentally appropriate assessment of young children. Illinois: ERIC/ EECE.

Hallahan, D. P. \& Kauffman, J. M. (1978). Exceptional children. Englewood Cliffs, NJ: Prentice Hall.

Hany, E. A. (1993). Methodological problems and issues concerning identification. En K. A. Heller, F. J. Mönks \& A. H. Passow (Eds.), International Handbook of Research and Develoment of Giftedness and Talent (pp. 209-232). Oxford: Pergamon Press.

Herskovitz, M. \& Gyarmathy, E. (1995). Gifted children early years by parental interview. En M. W. Katzko \& F. J. Mönks (Eds.), Nurturing talent. Individual needs and social ability. 
Meta-análisis sobre estudios de identificación y atención al talento.

The IV Conference of the European Council for High Ability. Assen, Holanda: Van Gorcum.

Humphreys, L. G. (1985). A conceptualization of intellectual giftedness. En F. D. Horowitz \& M. O’Brien (Eds.), The gifted and talented: Developmental perspectives. Washington, DC: American Psychological Association.

Janos, P. M. \& Robinson, N. M. (1985). Psychosocial development in intellectually gifted children. En F. E. Horowitz \& M. O’Brien (Eds.), The gifted and talented: Developmental perspectives. Washington, DC: American Psychological Association.

Keighley, R. (1984, abril). Utilising the gifted education policy in New South Wales. Comunicación presentada en la Conferencia de la Australian Association for the Education of the Gifted and Talented, Melbourne, Australia.

Lohman, D. (1992). Encouraging the development of fluid abilities in gifted students. En N. Colangelo, S. G. Assouline \& D. L. Ambroson (Eds.), Talent development: Proceedings from 1991 Henry and Jocelyn Wallace National Research Symposium on Talent Development (pp. 143-162). Unionville, NY: Trillium Press.

Meisels, F. \& Steele, D. (1991). The early childhood portfolio collection process. Ann Arbor, MI: Center for Human Growth and Development, Universidad de Michigan.

McLeod, Z. \& Kluckmann, I. (1985). A Trans-Canada survey of programming for gifted children. A preliminary report. Saskatoon, Canadá: Universidad de Saskatchewan/Institute of Child Guidance and Development.

McLeod, J. \& Cropley, A. (1989). Fostering academic excellence. Londres: Pergamon Press.

Mönks, F. J. (1992). Development of the gifted child: The issue of identification and programming. En F. J. Mönks \& W. Peters, Talent for the future (pp. 191-202). Maastricht; Holanda: ASSEN. 
Mooney, R. (1963). A conceptual model for integration from approaches to the identification of creative talent. En C. W. Taylor \& F. Barron (Eds.), Scientific creativity: Its recognition and development. Nueva York: Wiley \& Sons.

Passow, H. (1985). Education of the gifted and talented. En T.

Husen \& T. N. Postlethwaite (Eds.), International Encyclopedia of Education, Oxford: Pergamon Press.

Pedulla, J. J., Airasian, P. W. \& Manaus, G. (1980). Do teacher rating and standardised test results of students yield the same information? American Educational Research Journal, 17, 291-302.

Roe, A. (1952). The making of a scientist. Nueva York: Dodd, Mead.

Ruiz, C. (1991). Diferencias en las características de personalidad entre niños de ambos sexos bien dotados intelectualmente y aquellos de inteligencia normal. Lima: Universidad Nacional Mayor de San Marcos.

Silverman, L. K. (1993). The gifted individual. En L. K. Silverman (Ed.), Counseling the gifted and talented. Denver, CO: Love Publishing.

Stanley, J. C. (1984). Use of general and especific attitude measure in identification: Some principles and certain cautions. Gifted Child Quarterly, 28, 177-180.

Stephenson, M. (2000). Development and validation of the Stephenson Multigroup Acculturation Scale (SMAS). Psychological Assessment, 12, 77-88.

Sternberg, R. J. (1996). Successful intelligence. Nueva York: Simon \& Schuster.

Thorndike, R. L. \& Hagen, E. P. (1993). Cog At Form 5. Interpretive guide for teachers and counselors. Itasca, IL: Riverside Publishing.

Thorne, C. \& Blumen, S. (1996, agosto). Age, schooling and the Raven scores. Documento presentado en la XIII International Conference of Applied Psychology, Montreal, Canadá. 
Meta-análisis sobre estudios de identificación y atención al talento.

Torrance, E. P. (1979). The search for satori and creativity. Buffalo, NY: Bearly Limited.

Torrance, E. P. \& Ball, O. (1984). Torrance tests of creative thinking. Streamlined scoring workbook. Bensenville, IL: Scholastic Testing Service.

Urban, K. K. (1994, abril). Different models for describing, exploring, explaining, and nurturing creativity. Comunicación presentada en el Congreso Internacional Exploración y Desarrollo del Potencial Creativo, Pavia, Italia.

Urban, K. K. (1995). Test for Creative Thinking-Drawing Production. Frankfurt: Swets.

Urban, K. K. (1996). Test for creative thinking-drawing production. Frankfurt: Swets.

Urban, K. K. \& Jellen, H. G. (1994). Assessing creative potential via drawing production. The Test for Creative ThinkingDrawing Production (TCT-DP). En A. Cropley, K. K. Urban, H. Wagner \& W. Wieczerkowski (Eds.), Giftedness: $A$ continuing worldwide challenge (pp. 163-169). Nueva York: Trillium Press.

Yarborough, M. D. \& Johnson, L.W. (1983). Identifying the gifted: A theory-practice gap. Gifted Child Quarterly, 27, 135-138. 